\title{
Adsorbed liposome deformation studied with quartz crystal microbalance
}

\author{
Ilya Reviakine, ${ }^{1,2, a)}$ Marta Gallego, ${ }^{1}$ Diethelm Johannsmann, ${ }^{3}$ and Edurne Tellechea ${ }^{1, b}$ \\ ${ }^{1}$ CIC biomaGUNE, Paseo Miramón182, San Sebastián 20009, Spain \\ ${ }^{2}$ Department of Biochemistry and Molecular Biology, University of the Basque Country, 48940 Leioa, Spain \\ ${ }^{3}$ Institute of Physical Chemistry, Clausthal University of Technology, Arnold-Sommerfeld-Str. 4, D-38678 \\ Clausthal-Zellerfeld, Germany
}

(Received 1 December 2011; accepted 3 February 2012; published online 23 February 2012)

\begin{abstract}
Deformation of surface-adsorbed liposomes is an important parameter that governs the kinetics of their transformations, but one that is very difficult to measure in the case of nm-size liposomes. We investigate the deformation of dimyristoyl phosphatidyl choline liposomes by quartz crystal microbalance (QCM) as a function of temperature and show that it follows the dependence of this lipid's bending modulus on temperature, as expected from theoretical considerations. To corroborate our approach, we model QCM response from adsorbed liposomes by explicitly considering their shape and mechanical properties. ( 2012 American Institute of Physics. [http://dx.doi.org/10.1063/1.3687351]
\end{abstract}

\section{INTRODUCTION}

The behavior of small (30-100 nm) liposomes ${ }^{1}$ adsorbed at surfaces is complex and remains poorly understood..$^{2-5}$ It is important in the context of developing new materials with tailored surface biofunctionalities, especially those incorporating transmembrane proteins..$^{5-7}$ Liposomes also serve as models for complex biological systems and can be used to elucidate the principles underlying bio/non-bio interactions relevant to biocompatibility. ${ }^{8}$

Adsorbing liposomes are expected to deform, as shown schematically in Figure 1. ${ }^{9}$ The shape of the deformed liposome is governed by three contributions. On the one hand, deformation is driven by the adhesion energy that arises from the lipid-surface interactions and strives to maximize the area of contact between the liposome membrane and the surface. On the other hand, it is opposed by the cost of bending the lipid bilayer. Finally, osmotic pressure may favor the volume changes associated with the deformation or oppose them, depending on the direction of the osmotic gradient. ${ }^{9}$ Adhesion and bending contributions can be combined into a dimensionless parameter called reduced interaction potential $w=W R^{2} / \kappa$, where $W$ is the adhesion energy, $R$ is the radius of the free liposome, and $\kappa$ is the bilayer bending modulus. Shapes of liposomes for various values of the reduced potential and osmotic pressure have been calculated by Seifert et al. ${ }^{9-11}$

Experimentally, liposome deformation has been studied extensively using giant liposomes (giant unilamellar vesicles (GUVs)) in weak potentials. ${ }^{12}$ The results of these experiments are in good agreement with the theory. On the other hand, liposomes with sizes in the nm-range present a number of problems. From the theoretical point of view, the separation of length scales between the range of surface-lipid in-

\footnotetext{
a) Author to whom correspondence should be addressed. Electronic mail: IReviakine@ cicbiomagune.es.

b) Present address: FideNa, Edificio Jerónimo de Ayanz, C/Tajonar s/n, 31006 Pamplona, Navarra, Spain; and Instituto de Agrobiotecnología, Universidad Pública de Navarra-CSIC Gobierno Navarra, Campus Arrosadía, 31006 Pamplona, Navarra, Spain.
}

teractions (in $\mathrm{nm}$ ) and liposome size (in $\mu \mathrm{m}$ for the GUVs) is one of the key assumptions underlying adsorbed liposome shape calculations. ${ }^{9}$ Furthermore, nm-sized liposomes are already highly curved, with the radius of curvature that is comparable to the molecular size. Therefore, it is by no means guaranteed that the picture obtained with the GUVs can be transferred to them. Experimentally, deformation of nm-sized liposomes is much more difficult to study because of their small size. Only recently it was conclusively shown by cryo transmission electron microscopy (cryoTEM) that they actually deform upon adsorption-see Ref. 7 and Figure 2. CryoTEM allows deformed liposomes to be visualized directly. There are very few other studies discussing adsorbed liposome deformation. ${ }^{13-16}$ Liposome deformation in those studies is inferred indirectly via model-based analysis of acoustic (quartz crystal microbalance $(\mathrm{QCM}))^{13-15}$ or optical (dual polarization interferometery) ${ }^{16}$ measurements, and the results depend on the assumptions used in the models. Here, we present and validate a new method for measuring the deformation of nm-sized liposomes adsorbing on surfaces. Although it is based on deducing the height of surface-adsorbed liposomes (labeled as $h$ in Figure 1) from QCM ${ }^{17-21}$ measurements, it does not rely on a model. Previously, we showed that this method reliably and quantitatively yields the size of the adsorbed particles for stiff particles that do not deform upon adsorption; in that case, $h \sim 2 R$ (Figure 1(a)), where $R$ is the radius of particles in solution (e.g., measured with dynamic light scattering). ${ }^{22}$ Here, we validate this method for studying the deformation of the much softer particles that do deform when they adsorb to the surface.

\section{MATERIALS AND METHODS}

\section{Materials}

Chemicals used were $\geq 99.0 \%$ purity and purchased from Sigma-Aldrich (Madrid, Spain) unless otherwise indicated. Chloroform stabilized with ethanol was purchased from Scharlab (Barcelona, Spain). Lipids-1,2-dioleoyl- 
(a)

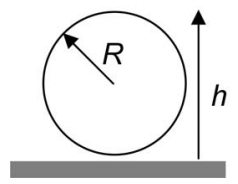

(b)

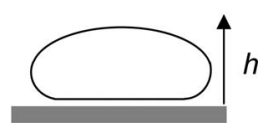

FIG. 1. Schematic illustration of adsorbed liposome deformation. (a) Undeformed (spherical) liposome. Its height $h$ is equal to its diameter, $2 R, h=2 R$, where $R$ is the radius of liposome in solution. (b) Deformed (dome-shaped) liposome. The height $h$ of a deformed liposome above the surface will be smaller than that of the undeformed one of the same size. The parameter we measure in this study is $h$, adsorbed liposome height.

sn-glycero-3-phosphocholine (DOPC), 1,2-dimyristoyl-snglycero-3-phosphocholine (DMPC), and 1,2-dipalmitoyl-snglycero-3-phosphocholine (DPPC)—were purchased from Avanti Polar Lipids, Inc. (Alabama, USA), in powder form and stored at $-20^{\circ} \mathrm{C}$ until used. Titania-coated quartz crystals $(5 \mathrm{MHz})$ were purchased from Q-Sense AB (Västra Frölunda, Sweden). Water was purified with a Diamond UV water purification system (Branstead International, Iowa, USA). Two buffers were used in this study: $10 \mathrm{mM}$ HEPES, $150 \mathrm{mM}$ $\mathrm{NaCl}, \mathrm{pH} 7.4$, and the same buffer containing $2 \mathrm{mM} \mathrm{Ca}$, referred to as Ca-free and Ca buffer, respectively. $300 \mathrm{~nm}$ silica particles used in CryoTEM experiments were purchased from Kisker Biotech GmbH \& Co. KG (Steinfurt, Germany) and stored at $4{ }^{\circ} \mathrm{C}$. Immediately before use, the particles were diluted in the Ca-free buffer. Their Z-average size, measured by DLS, was $294 \pm 22 \mathrm{~nm}$.

\section{Methods}

\section{Liposome preparation and characterization}

DMPC liposomes used in quartz crystal microbalance experiments were prepared by extrusion through $100 \mathrm{~nm}$ pore diameter filters in a hand-held LipoFast extruder (Avestin,

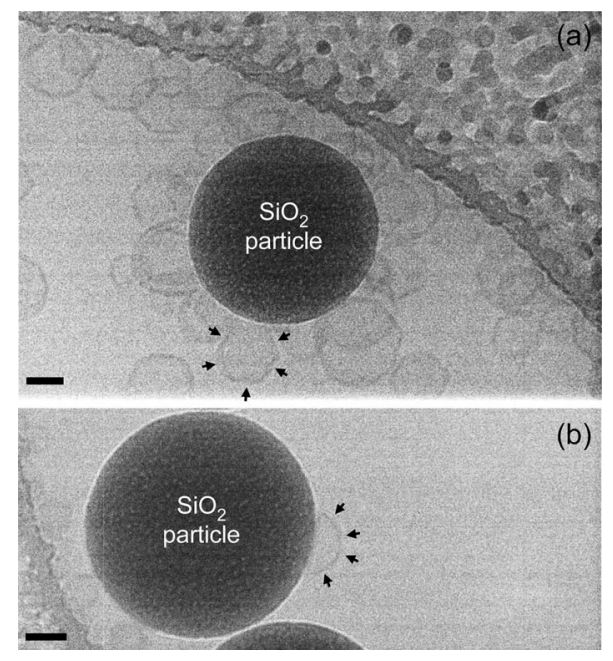

FIG. 2. CryoTEM images of (a) liposomes made of lipid in the gel phase (dipalmitoyl phosphatidyl choline (DPPC)) and (b) liposomes made of a lipid in the fluid phase (dioleoyl phosphatidyl choline (DOPC)) adsorbed on 300 $\mathrm{nm}$ silica particles at room temperature. Liposomes adhering to the particles are indicated with the black arrowheads. Note the polygonal shape of DPPC liposomes in (a) and the dome shape of the DOPC liposome in (b). The scale bar is $100 \mathrm{~nm}$.
Ottawa, Canada) as described previously. ${ }^{3,22,23}$ DPPC liposomes used in CryoTEM experiments were prepared by extrusion through $50 \mathrm{~nm}$ diameter filters, while DOPC ones were prepared by sonication using a Branson Ultrasonic 450 tip sonicator (Danbury, USA) set at 30\% duty cycle and at an output power of 3 . Sonication was done at $0{ }^{\circ} \mathrm{C}$ under nitrogen atmosphere. Freshly sonicated liposomes were centrifuged under argon atmosphere for $3 \mathrm{~h}$ at $65000 \times \mathrm{g}$ in a Sigma 3K30 centrifuge (Sigma Centrifuges, Shropshire, UK).

Liposomes were characterized by dynamic light scattering with a Zetasizer Nano series instrument (Malvern, Worcestershire, UK). Sonicated DOPC liposomes exhibited two peaks, with diameters $47 \pm 6$ and $115 \pm 12 \mathrm{~nm}$ by intensity. ${ }^{24}$ Extruded liposomes exhibited one peak. The Zaverage size of DPPC liposomes was $\sim 91 \mathrm{~nm}$ at $25^{\circ} \mathrm{C}$. The size of DMPC liposomes was measured as a function of temperature. The results are shown in Figure 4(b).

\section{Quartz crystal microbalance experiments}

Temperature-dependent QCM experiments were performed with DMPC liposomes in Ca buffer as described in Tellechea et al. ${ }^{22}$ Lipid concentration was $0.25 \mathrm{mg} / \mathrm{ml}$.

\section{CrYoTEM}

CryoTEM experiments were performed in a JEM-1230 transmission electron microscope equipped with $\mathrm{a} \mathrm{LaB}_{6}$ cathode running at $120 \mathrm{kV}$ and an ORIOUS SC1000 $4008 \times 2672$ pixels CCD camera (Gatan, UK; Abingdon Oxon, UK). Samples were prepared using Quantifoil grids (200 square mesh, $2 \mu \mathrm{m}$ circular holes, period $3 \mu \mathrm{m}$ ) from SPI (West Chester, USA). The grids were treated in a chloroform atmosphere overnight and vacuum-dried for $1 \mathrm{~h}$ afterwards. They were rendered negatively charged by glow discharge treatment in a MED 020 modular high vacuum coating system (BAL-TEC AG, Fuerstentum, Liechtenstein) for $15 \mathrm{~s}$ at $2.0 \times 10^{-1} \mathrm{~atm}$ and $10 \mathrm{~mA}$ and mounted in the VITROBOT (FEI Europe, Eindhoven, The Netherlands). The $3.4 \mu 1$ of $2 \mathrm{mg} / \mathrm{ml}$ DOPC or DPPC liposome solution in Ca-free buffer was pipetted into the grid, followed by $0.2 \mu \mathrm{l}$ of $2 \mathrm{mg} / \mathrm{ml}$ silica particle solution prepared in the same buffer. Final PC:Si ratio was 17:1. PC:Si by weight. DOPC:Si samples were vitrified immediately after the addition of liposomes, while the DPPC:Si suspensions were incubated at room temperature and $100 \%$ humidity for 3 min before vitrification. Vitrified grids were stored and transferred under liquid nitrogen, installed in the electron microscope, and imaged in a low-dose mode at $30000 \times$ magnification. The images are shown in Figure 2.

\section{RESULTS AND DISCUSSION}

As a model system for studying adsorbed liposome deformation, we use dimyristoyl phosphatidyl choline (DMPC) liposomes adsorbing on $\mathrm{TiO}_{2}$. DMPC has the main (gel-tofluid) transition at $\sim 24^{\circ} \mathrm{C}$ (Ref. 25), making it convenient to study liposome adsorption above and below this temperature. At temperatures below the main transition temperature, the lipid bilayer is stiff $\left(\kappa\right.$ is of the order of $10 \times 10^{-19} \mathrm{~J}$ for both dipalmitoyl phosphatidyl choline (DPPC) ${ }^{26}$ and DMPC 
(Ref. 27) in the $L_{\beta^{\prime}}$ phase). At temperatures above the transition temperature, the bilayer is in the fluid phase, and $\kappa$ is of the order of $1 \times 10^{-19} \mathrm{~J}^{26,28,29}$ As we have seen above, bilayer bending opposes deformation. We therefore expect that liposomes made of a lipid in the fluid phase (above the transition temperature) would deform to a greater extent than those made of a lipid in the gel phase (Figure 1). Indeed, in Figure 2 , we show that this expectation is correct. CryoTEM images of liposomes made of a lipid in the gel phase (DPPC) show liposomes that maintain their polygonal shape upon adsorption and do not significantly deform (Figure 2(a)). We have also previously shown that the height of DPPC liposomes adsorbed on the surface at room temperature $\left(\sim 20^{\circ} \mathrm{C}\right.$ below its transition temperature) corresponds to their diameter in solution. ${ }^{22}$ On the other hand, liposomes made of a lipid in its fluid phase (DOPC) show deformed, dome-shaped structures (Figure 2(b)). This shape has been observed previously ${ }^{7}$ and is qualitatively consistent with theoretical predictions. ${ }^{9}$

We follow the adsorption of $\sim 90 \mathrm{~nm}$ (diameter) DMPC liposomes on $\mathrm{TiO}_{2}$ with $\mathrm{QCM}^{17-21}$ at different temperatures. QCM works by monitoring the resonance frequency, $f_{n}$, and the bandwidth, $\Gamma_{n}$, of a quartz oscillator as material adsorbs to its surface. Bandwidth $\Gamma$, used throughout this work, is equivalent to the dissipation $D$ used by the QCM-D community: ${ }^{19}$ $D=2 \Gamma_{n} / f_{n}$, where $f_{n}$ is the resonance frequency of the crystal and $n$ is the overtone order. Measurements are done on several overtones; $n$ is the overtone order and $f_{n} \sim n f_{\mathrm{F}}$, where $f_{\mathrm{F}}$ is the fundamental frequency of the crystal. Our crystals have the fundamental frequency $f_{\mathrm{F}} \sim 5 \mathrm{MHz}$, and we perform the measurements on overtones $n=3,5,7,9$, and 11 (between $\sim 15$ and $55 \mathrm{MHz}$ ). The results are plotted as differences relative to the frequency and bandwidth of the bare crystal, scaled by the overtone order: $\Delta F / n=\left(f_{n}-f_{n \text {, bare }}\right) / n$ and $\Delta \Gamma / n=\left(\Gamma_{n}\right.$ $-\Gamma_{n}$,bare $) / n$, where $f_{n}$, bare and $\Gamma_{n \text {,bare }}$ are the resonance frequency and bandwidth of the bare crystal in liquid measured before adding the liposomes.

Typical results of such measurements are shown in Figures 3(a) and 3(c) for experiments performed at $10^{\circ} \mathrm{C}$ (below the transition) and $32^{\circ} \mathrm{C}$ (above the transition), respectively. In both cases, adsorption of liposomes causes a decrease in the frequency and an increase in the bandwidth, until both parameters saturate at their asymptotic values. This saturation behavior has been observed in numerous previous studies of liposome adsorption on surfaces., ${ }^{4,13,15}$ The asymptotic shifts are larger at $10{ }^{\circ} \mathrm{C}$ than at $32^{\circ} \mathrm{C}$. This is consistent with our previous findings, showing that the gelphase (stiffer) liposomes elicit larger asymptotic shifts than the fluid-phase ones of similar size. ${ }^{15}$ Figure S1 in the supplementary material shows the dependence of the asymptotic shifts on temperature. ${ }^{31}$

It is important to realize that QCM does not deal with individual liposomes in the way TEM (Figure 2) does. Instead, it deals with films composed of many liposomes adsorbed on the surface side by side (Figures 3(b) and 3(d), insets). The thickness of such a film could be directly determined from the frequency shifts using the Sauerbrey relationship ${ }^{17}$ ( $h=-\Delta F / n \times C / \rho$, where $C=18 \mathrm{ng} / \mathrm{cm}^{2} / \mathrm{Hz}$ and $\rho=1 \mathrm{~g} / \mathrm{cm}^{3}$ is the density of the film) if the bandwidth shifts $\Delta \Gamma_{n}$ were close to zero and the frequency shifts were the same on all
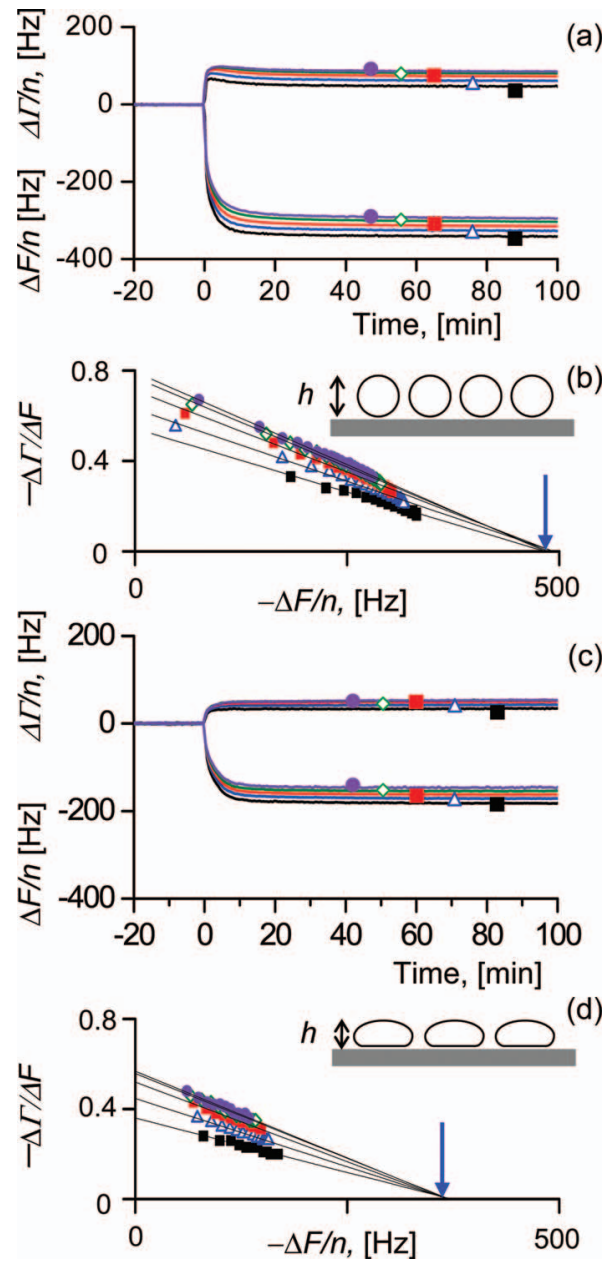

FIG. 3. Adsorption of DMPC liposomes on $\mathrm{TiO}_{2}$ as a function of temperature studied by QCM. The raw data (changes in the frequency $\Delta F / n$, and bandwidth (dissipation), $\Delta G / n$ as a function of time), and the corresponding $-\Delta G / \Delta F$ vs. $\Delta F / n$ plots used to extract the equivalent film thicknesses, are shown in (a), (b) and (c), (d) for the experiments done at $10^{\circ} \mathrm{C}$ and $32^{\circ} \mathrm{C}$, respectively. Overtones $n=3$ (black solid squares), 5 (blue open triangles), 7 (red solid squares), 9 (green open circles), and 11 (purple solid circles) are shown. In (a) and (c), the frequency shifts are negative, while the bandwidth shifts are positive. Blue arrowheads in (b) and (d) point to the common intercept with the $\mathrm{X}$ axis of the extrapolated plots used to extract equivalent film thickness $h$. Insets schematically show a film of undeformed liposomes (b) and a film of deformed liposomes (d). Note the maximum in the bandwidth vs. time plot in panel (a).

overtones $\left(\Delta F_{n} / n \sim\right.$ const $)$. In the case of liposomes adsorbed on the surface (Figure 3), as in many other cases, ${ }^{20}$ these two conditions are not satisfied and the QCM signals contain contributions from the thickness of the adsorbed film, which depends on the height of the adsorbed liposomes as well as on the surface coverage (number of liposomes adsorbed per unit area), from the strength of the liposome-surface interactions, and also possibly from the lipid bilayer stiffness. ${ }^{20,22,32-34}$ To extract the height of adsorbed liposomes, we plot the ratio of the two measured parameters, $-\Delta \Gamma / \Delta F$, as a function of the frequency shift $-\Delta F / n .^{22}$ These plots are shown in Figures 3 (b) and 3 (d) for the measurements done at 10 and $32^{\circ} \mathrm{C}$, respectively. Measurements done on several overtones extrapolate to a common intercept with the $\mathrm{X}$ axis (blue arrowheads in Figures 3(b) and 3(d)), where the $\Delta \Gamma$ is zero, yielding a unique value of $-\Delta F_{n} / n$ that can be plugged into the 


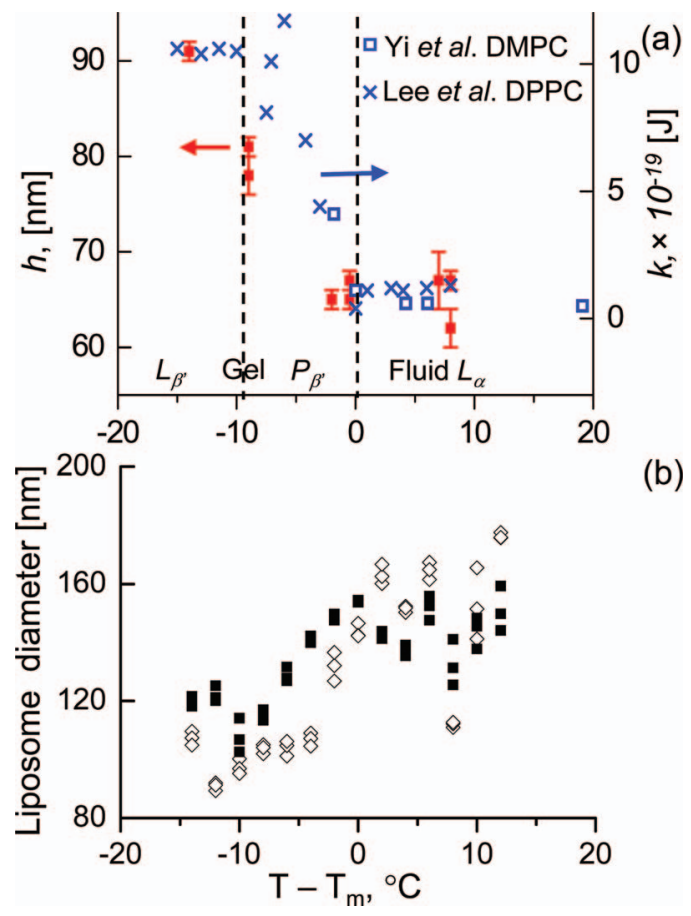

FIG. 4. (a) The plot of the equivalent layer thickness $h$ (red filled squares, left axis) for the DMPC liposomes adsorbed on $\mathrm{TiO}_{2}$, and the bilayer bending modulus $\kappa$ for DPPC (Ref. 26) and for DMPC (Ref. 35) (blue crosses, blue open squares, right axis) are plotted as a function of reduced temperature, $T-T_{m}$, where $T_{m}$ is the main transition temperature of the appropriate lipid $\left(\sim 24^{\circ} \mathrm{C}\right.$ for DMPC (Ref. 25$)$ and $\sim 42^{\circ} \mathrm{C}$ for DPPC (Ref. $25)$ ). Three different phases of the lipids are indicated along the reduced temperature axis: the high-temperature fluid phase $\left(L_{\alpha}\right)$, and the two gel phases, $P_{\beta^{\prime}}$ and $L_{\beta^{\prime}}$ that occur below the main transition. ${ }^{25}$ (b) Z-average size of DMPC liposomes in solution as a function of temperature measured with dynamic light scattering. Open symbols: increasing temperature. Filled symbols: decreasing temperature.

Sauerbrey relationship to give an equivalent film thickness $h$. In the following, we demonstrate that this equivalent film thickness can be identified with the height of the adsorbed liposomes as defined in Figure 1.

First, it is evident from Figures 3(b) and 3(d) that this equivalent thickness is smaller in the case of liposomes adsorbing above the transition temperature $\left(32^{\circ} \mathrm{C}\right)$ than in the case of liposomes adsorbing below the transition temperature $\left(10^{\circ} \mathrm{C}\right)$, indicating that liposomes adsorbing at a higher temperature (in fluid phase) are deformed to a greater extent. This is consistent with what we expect based on the CryoTEM results (Figure 2).

Second, in Figure 4(a), the equivalent film thickness $h$ is plotted as a function of temperature together with the bilayer bending modulus for DPPC measured by Lee $\mathrm{et} \mathrm{al.}{ }^{26}$ and for DMPC measured by Yi et al. ${ }^{35}$ There is a clear correspondence between the behavior of the equivalent thickness and the bending modulus as a function of temperature: below the main transition temperature, when the lipid is in the $P_{\beta^{\prime}}$ phase, the bending modulus increases with decreasing temperature. ${ }^{26,29,35,36}$ There is a corresponding increase in the equivalent thickness extracted from the QCM data. Identifying the equivalent thickness with the height of the adsorbed liposomes, we explain the observed correspondence in terms of the effect of the bending modulus on the adsorbed liposome deformation: higher bending modulus (stiffer lipo- somes) leads to smaller deformation and larger equivalent thickness.

The bending moduli shown in Figure 4(a) deserve a comment. The temperature dependence of the bending modulus in the $P_{\beta^{\prime}}$ phase has been reported by several investigators for DPPC and for DMPC. ${ }^{26,29,35,36}$ The DPPC data illustrate this trend best; therefore, we compare our results with these data. The absolute values of the bending moduli in the gel phase vary, however: the values reported by Dimova et al. ${ }^{36}$ for DMPC reach $\sim 25-35 \times 10^{-19} \mathrm{~J}$ at $\sim 19^{\circ} \mathrm{C}$, which is significantly higher than those reported by other authors. ${ }^{27,35}$ For instance, Tristam-Nagle et al. ${ }^{27}$ give $\kappa \sim 10 \times 10^{-19} \mathrm{~J}$ for the gel phase of DMPC, which is similar to the value obtained for DPPC in the same phase, as shown in Figure 4(a). ${ }^{26}$

Third, we show in Figure 4(b) that the size of DMPC liposomes in solution (not adsorbed to a surface) increases with temperature. The equivalent thickness $h$, on the other hand, decreases with temperature (Figure 4(a)). Therefore, the equivalent thickness determined from the QCM measurements reflects the height of the surface-adsorbed liposomes above the surface, and not the size of the liposomes in solution.

Finally, to corroborate our way of assessing adsorbed liposome heights from $-\Delta \Gamma / \Delta F$ vs. $-\Delta F / n$ plots, we performed finite element method (FEM) calculations that we have previously described and validated. ${ }^{22,32,34}$ FEM allows the frequency and bandwidth shifts due the stress exerted at the crystal surface by the adsorbed particles that are moved through the surrounding liquid by QCM crystal's oscillatory motion to be calculated via the small load approximation. ${ }^{21}$ Stress is calculated from the velocity fields obtained numerically using COMSOL's Incompressible Navier-Stokes Module. Adsorbed liposomes were modeled as truncated ellipsoidal shells of thickness $t=5 \mathrm{~nm}$, corresponding to the thickness of a lipid bilayer, complex shear modulus $G$ $=|\mathrm{G}| \cdot(\cos \delta+i \cdot \sin \delta)$, where $\delta$ is the loss angle such that $|\mathrm{G}|=12 \kappa / t^{3} ; \delta$ and $\kappa$ were varied, and shapes that are shown in Figure 5(a) taken from Ref. 10. Further details are given in the supplementary material. ${ }^{31}$ The resulting $-\Delta \Gamma / \Delta F$ vs. $-\Delta F / n$ plots are shown in Figure $5(\mathrm{~b})$ for $\kappa=85 \times 10^{-19} \mathrm{~J}$ and $\delta=45^{\circ}$. The observed decrease in the $-\Delta \Gamma / \Delta F$ with coverage and smaller values of the ratio for liposomes that are more deformed are qualitatively reproduced. The extrapolation of the plots to $-\Delta \Gamma=0$ yields $\Delta F / n=-166 \mathrm{~Hz},-232$ $\mathrm{Hz}$, and $-300 \mathrm{~Hz}$ leading to the equivalent thickness $h=28$, 42 , and $49 \mathrm{~nm}$ for adsorbed liposome heights of 30,43 , and $54 \mathrm{~nm}$, respectively. This is a very convincing correlation.

It is interesting to note that the FEM calculations best reproduce experimental results for rather high values of $\kappa$ $\left(85 \times 10^{-19} \mathrm{~J}\right.$, Figure $\left.5(\mathrm{~b})\right)$, indicating that at $\mathrm{MHz}$ frequencies, the lipid membrane appears significantly stiffer than at zero frequency. Indeed, the bending stiffness should display viscoelastic dispersion like any other mechanical property in soft matter experiments, and it is not reasonable to expect the values determined from low-frequency experiments on giant liposomes ${ }^{26,28,29,36}$ to be applicable at the time scales probed by the $\mathrm{MHz}$ frequencies of QCM. $\kappa$ is expected to be much higher at $\mathrm{MHz}$ frequencies than at low frequencies, and this can be corroborated by comparing the outcome of FEM 


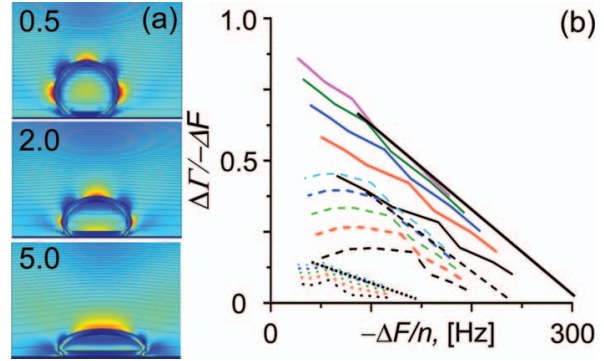

FIG. 5. (a) Shapes of the adsorbed liposomes for three different values of the reduced potential $w=0.5,2.0$, and 5.0, in the absence of an osmotic gradient, were taken from Ref. 10. The magnitudes of the reduced potential are indicated in each of the panels. Color represents local dissipation rate per unit volume. Brighter areas correspond to higher rates. (b) $\Delta \Gamma / \Delta F$ vs. $-\Delta F / n$ plots calculated for $R=25 \mathrm{~nm}, \kappa=85 \times 10^{-19} \mathrm{~J}$, and $\delta=45^{\circ}$ for the three shapes shown in (a). Full lines: $w=0.5$. Dashed lines: $w=2.0$. Dotted lines: $w=5.0$. Extrapolation, as shown in Fig. 3, is also schematically indicated.

calculations with our experiments. Experimentally, there is often a maximum in bandwidth at intermediate coverages, such as the one appearing in Figure 3(a), or the one that appears in our earlier publication, where it was observed with fluid-phase (DOPC) liposomes. ${ }^{15}$ We have discussed the reasons behind the occurrence of such maxima at length in Ref. 32. They arise from the "rocking and rolling" motion of the adsorbed particles at the surface. This motion induces a large amount of dissipation in the nearby liquid. Dissipation increases with the number of particles, but as the coverage increases, the hydrodynamic interaction between neighbors increasingly prevents this type of motion, thereby reducing the dissipation in the film. Importantly, this mechanism requires the adsorbate to be sufficiently stiff-otherwise, it is deformed, rather than rotated. The fact that the experiment shows this maximum (cf. Figure 3(a)) indicates that liposomes appear stiff at $\mathrm{MHz}$ frequencies. We can only reproduce this maximum if we chose the correspondingly high bending stiffness (about 10-50 times the static bending modulus). The deformation, which occurs on a significantly longer time scales than QCM oscillations, is, of course, governed by the static bending modulus.

In conclusion, we deduced the height of nm-sized liposomes adsorbed on $\mathrm{TiO}_{2}$ surface from QCM data. As expected from theoretical considerations, it correlates with the bilayer bending modulus, with softer liposomes showing smaller heights (deforming to a greater extent). To corroborate our measurements, we modeled QCM response from adsorbed liposomes by explicitly considering their shape and mechanical properties. Our method of measuring adsorbed liposome deformation opens new possibilities for studying liposomesurface interactions. Effects of parameters, such as liposome composition, surface properties, and osmotic pressure, can now be systematically examined and compared with theoretical predictions.

\section{ACKNOWLEDGMENTS}

We would like to acknowledge the funding from the Department of Industry of the Basque Government (Pro- gram ETORTEK) and from the Spanish Ministry of Science and Innovation (MICINN, ref. CTQ2009-11245) to I.R. and thank David Gil Carton from the CIC bioGUNE Electron Microscopy facility (Derio, Spain) for assistance with the use of their CryoTEM equipment.

${ }^{1}$ A. D. Bangham, Chem. Phys. Lipids 64(1-3), 275 (1993); D. D. Lasic and D. Papahadjopoulos, Science 267(5202), 1275 (1995).

${ }^{2}$ P. Nollert, H. Kiefer, and F. Jahnig, Biophys. J. 69(4), 1447 (1995); P. S. Cremer and S. G. Boxer, J. Phys. Chem. B 103(13), 2554 (1999).

${ }^{3}$ I. Reviakine and A. Brisson, Langmuir 16(4), 1806 (2000).

${ }^{4}$ C. A. Keller and B. Kasemo, Biophys. J. 75(3), 1397 (1998); C. A. Keller, K. Glasmastar, V. P. Zhdanov, and B. Kasemo, Phys. Rev. Lett. 84(23), $5443(2000)$.

${ }^{5}$ R. P. Richter, R. Berat, and A. R. Brisson, Langmuir 22(8), 3497 (2006).

${ }^{6}$ E. Sackmann, Science 271(5245), 43 (1996); S. Trepout, S. Mornet, H. Benabdelhak, A. Ducruix, A. R. Brisson, and O. Lambert, Langmuir 23(5), 2647 (2007).

${ }^{7}$ S. Mornet, O. Lambert, E. Duguet, and A. Brisson, Nano Lett. 5(2), 281 (2005).

${ }^{8}$ B. Kasemo, Surf. Sci. 500(1-3), 656 (2002); F. F. Rossetti, M. Bally, R. Michel, M. Textor, and I. Reviakine, Langmuir 21, 6443 (2005).

${ }^{9}$ U. Seifert, Adv. Phys. 46(1), 13 (1997).

${ }^{10}$ U. Seifert, Phys. Rev. A 43(12), 6803 (1991).

${ }^{11}$ U. Seifert and R. Lipowsky, Phys. Rev. A 42(8), 4768 (1990).

${ }^{12}$ T. Gruhn, T. Franke, R. Dimova, and R. Lipowsky, Langmuir 23(10), 5423 (2007); A. S. Smith, E. Sackmann, and U. Seifert, Europhys. Lett. 64(2), 281 (2003).

${ }^{13}$ E. Reimhult, F. Hook, and B. Kasemo, Langmuir 19(5), 1681 (2003).

${ }^{14}$ E. Reimhult, F. Hook, and B. Kasemo, J. Chem. Phys. 117(16), 7401 (2002).

${ }^{15}$ I. Reviakine, F. F. Rossetti, A. N. Morozov, and M. Textor, J. Chem. Phys. 122(20), 204711 (2005).

${ }^{16}$ T. R. Khan, H. M. Grandin, A. Mashaghi, M. Textor, E. Reimhult, and I. Reviakine, Biointerphases 3(2), FA90 (2008).

${ }^{17}$ G. Sauerbrey, Z. Phys. 155, 206 (1959).

${ }^{18}$ R. Beck, U. Pittermann, and K. G. Weil, Ber. Bunsenges. Phys. Chem. Chem. Phys. 92(11), 1363 (1988).

${ }^{19}$ M. Rodahl, F. Hook, A. Krozer, P. Brzezinski, and B. Kasemo, Rev. Sci. Instrum. 66(7), 3924 (1995).

${ }^{20}$ I. Reviakine, D. Johannsmann, and R. P. Richter, Anal. Chem. 83(23), 8838 (2011).

${ }^{21}$ D. Johannsmann, Phys. Chem. Chem. Phys. 10, 4516 (2008).

${ }^{22}$ E. Tellechea, D. Johannsmann, N. F. Steinmetz, R. P. Richter, and I. Reviakine, Langmuir 25(9), 5177 (2009).

${ }^{23}$ R. C. Macdonald, R. I. Macdonald, B. P. M. Menco, K. Takeshita, N. K. Subbarao, and L. R. Hu, Biochim. Biophys. Acta 1061(2), 297 (1991).

${ }^{24}$ C. H. Huang, Biochemistry 8(1), 344 (1969).

${ }^{25}$ A. Blume, Thermochim. Acta 193, 299 (1991).

${ }^{26}$ C. H. Lee, W. C. Lin, and J. P. Wang, Phys. Rev. E 64, 020901 (2001).

${ }^{27}$ S. Tristam-Nagle, Y. Liu, J. Legleiter, and J. F. Nagle, Biophys. J. 83, 3324 (2002).

${ }^{28}$ W. Rawicz, K. C. Olbrich, T. McIntosh, D. Needham, and E. Evans, Biophys. J. 79(1), 328 (2000).

${ }^{29}$ P. Méléard, C. Gerbeaud, T. Pott, L. Fernandez-Puente, I. Bivas, M. D. Mitov, J. Dufourcq, and P. Bothorel, Biophys. J. 72(6), 2616 (1997).

${ }^{30}$ R. Richter, A. Mukhopadhyay, and A. Brisson, Biophys. J. 85(5), 3035 (2003); B. Seantier, C. Breffa, O. Felix, and G. Decher, J. Phys. Chem. B 109(46), 21755 (2005).

${ }^{31}$ See supplementary material at http://dx.doi.org/10.1063/1.3687351 for the dependence of the asymptotic frequency and bandwidth shifts on temperature and for the technical details of the FEM calculations.

${ }^{32}$ D. Johannsmann, I. Reviakine, and R. P. Richter, Anal. Chem. 81(19), 8167 (2009).

${ }^{33}$ E. Rojas, M. Gallego, and I. Reviakine, Anal. Chem. 80(23), 8982 (2008).

${ }^{34}$ D. Johannsmann, I. Reviakine, E. Rojas, and M. Gallego, Anal. Chem. 80(23), 8891 (2008).

${ }^{35}$ Z. Yi, M. Nagao, and D. P. Bossev, J. Phys. Condens. Matter 21(15) (2009).

${ }^{36}$ R. Dimova, B. Pouligny, and C. Dietrich, Biophys. J. 79(1), 340 (2000). 